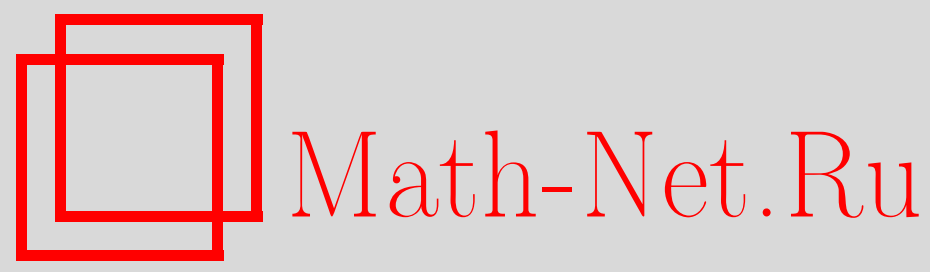

Я. В. Микитюк, М. Н. Шеремета, О. М. Сумык, О функциях, двойственных по Юнгу, и поведении максимальных членов производных ряда Дирихле, Матем. заметки, 2001, том 69, выпуск $1,74-81$

DOI: https://doi.org/10.4213/mzm484

Использование Общероссийского математического портала Math-Net.Ru подразумевает, что вы прочитали и согласны с пользовательским соглашением http://www.mathnet.ru/rus/agreement

Параметры загрузки:

IP : 3.85 .73 .92

26 апреля 2023 г., 10:40:15

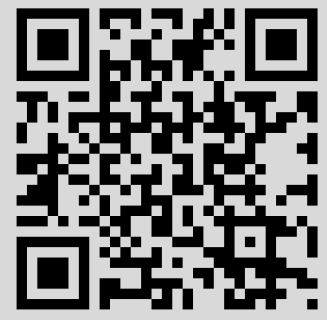


УДК 517.537 .2

\title{
О ФУНКЦИЯХ, ДВОЙСТВЕННЫХ ПО ЮНГУ, И ПОВЕДЕНИИ МАКСИМАЛЬНЫХ ЧЛЕНОВ ПРОИЗВОДНЫХ РЯДА ДИРИХЛЕ
}

\author{
Я.В. Микитюк, О.М. Сумык, М.Н. Шеремета
}

Из доказанного более общего результата о двойственных по Юнгу функциях получен критерий роста максимального члена ряда Дирихле с положительными показателями в терминах поведения максимальных членов последовательных производных этого ряда.

Библиография: 5 названий.

1. Введение. Пусть $1 \leqslant \lambda_{1}<\lambda_{n} \uparrow \infty, n \rightarrow \infty$, а ряд Дирихле

$$
\sum_{n=1}^{\infty} a_{n} \exp \left\{s \lambda_{n}\right\}, \quad s=\sigma+i t
$$

такой, что

$$
\varliminf_{n \rightarrow \infty} \frac{1}{\lambda_{n}} \ln \frac{1}{\left|a_{n}\right|}=A \in(-\infty,+\infty] .
$$

Легко видеть, что абсцисса абсолютной сходимости ряда (1) $\sigma_{a} \leqslant A$. Действительно, для любого $\sigma>A$ существует последовательность $\left(n_{k}\right) \uparrow \infty, k \rightarrow \infty$, такая, что

$$
\frac{1}{\lambda_{n}} \ln \frac{1}{\left|a_{n}\right|} \leqslant \sigma, \quad n=n_{k}
$$

т.е. $\left|a_{n}\right| \exp \left\{\sigma \lambda_{n}\right\} \geqslant 1, n=n_{k}$, и $\sum_{n=1}^{\infty}\left|a_{n}\right| \exp \left\{\sigma \lambda_{n}\right\}=+\infty$. Если не накладьвать никаких ограничений на показатели $\lambda_{n}$, то может случиться даже так, что $A=+\infty$ и $\sigma_{a}=-\infty$. Примером такого ряда Дирихле может служить ряд (1) с коэффициентами $a_{n}=\exp \{-\sqrt{\ln n}\}$ и показателями $\lambda_{n}=\ln \ln n, n \geqslant 3$.

Пусть $\mu(\sigma)=\max \left\{\left|a_{n}\right| \exp \left\{\sigma \lambda_{n}\right\}: n \geqslant 1\right\}-$ максимальньй член ряда (1). В силу (2) для каждого $\sigma<A$ максимальньй член существует, так как $\left|a_{n}\right| \exp \left\{\sigma \lambda_{n}\right\} \rightarrow 0$, $n \rightarrow \infty$, для таких $\sigma$. Если $\sigma_{a}=+\infty$ или $\sigma_{a} \in(-\infty,+\infty)$ и $\ln n=o\left(\lambda_{n}\right), n \rightarrow \infty$, то $\sigma_{a}=A$, сумма $F(s)$ ряда (1) является аналитической в $\{s: \operatorname{Re} s<A\}$ функцией и $\mu(\sigma, F)=\mu(\sigma), \sigma<A$. Если теперь $F^{(k)}-k$-я производная функции $F$, то

$$
\mu\left(\sigma, F^{(k)}\right)=\max \left\{\left|a_{n}\right| \lambda_{n}^{k} \exp \left\{\sigma \lambda_{n}\right\}: n \geqslant 1\right\}
$$

(C) Я.В. Микитюк, О. М. Сумык, М.Н. ШереметА 
- максимальньй член соответствующего ряда Дирихле. Мы сохраним это обозначение и в случае, когда $\sigma_{a}<A$.

В [1] доказано, что для любого фиксированного $\sigma<A$ последовательность $\left(\mu\left(\sigma, F^{(k)}\right)\right)$ неубывающая к $\infty$ и $\ln \mu\left(\sigma, F^{(k)}\right) \leqslant(1+o(1)) k \ln k$ при $k \rightarrow \infty$. Здесь мы установим связь между ростом $\ln \mu(\sigma)$ при $\sigma \uparrow A$ и поведением последовательности $\left(\mu\left(\sigma, F^{(k)}\right)\right)$.

Обозначим через $\Omega(A)$ клас положительных неограниченных на $(-\infty, A)$ функций $\Phi$ таких, что их производные $\Phi^{\prime}$ непрерьвные положительные и возрастающие к $+\infty$ на $(-\infty, A)$. Заметим, что если $\Phi \in \Omega(A), A \in(-\infty,+\infty]$, то $\Phi$ вьпуклая на $(-\infty, A)$, $\Phi(x) \rightarrow C \geqslant 0(x \rightarrow-\infty)$ и $\Phi^{\prime}(x) \rightarrow 0(x \rightarrow-\infty)$, ибо если $\Phi^{\prime}(x) \rightarrow k>0(x \rightarrow-\infty)$, то $\Phi(x) \sim k x(x \rightarrow-\infty)$, что невозможно. Как и в [2], функцию $\Psi(x)=x-\Phi(x) / \Phi^{\prime}(x)$ будем называть ассоииированной $c \Phi$ по Ньютону. Очевидно, что функция $\Psi$ непрерьвная на $(-\infty, A)$, если $\Phi \in \Omega(A)$. В [2] показано, что она возрастает к $A$ на $(-\infty, A)$. В дальнейшем через $\Psi^{-1}$ будем обозначать функцию, обратную к $\Psi$. Ясно, что функция $\Psi^{-1}$ непрерьвная и возрастает к $A$ на $(-\infty, A)$.

Справедлива следующая

ТЕорема 1. Пусть функиия $\Phi \in \Omega(A)$ удовлетворяет условиям:

a) $\Phi^{\prime}(\sigma)=O\left(\Phi^{\prime}(\Psi(\sigma))\right), \sigma \uparrow A$;

б) $\Phi^{\prime}\left(\sigma+\frac{1}{\Phi^{\prime}(\sigma)}\right)=O\left(\Phi^{\prime}(\sigma)\right), \sigma \uparrow A$;

в) $\Phi^{\prime}(\sigma)>1 /(A-\sigma), \sigma<A$.

Тогда для того чтобы $\ln \mu(\sigma)=O(\Phi(\sigma)), \sigma \uparrow A$, необходимо и достаточно, чтоби существовало число $N \in \mathbb{Z}_{+}$такое, что

$$
\frac{\mu\left(\sigma, F^{(n)}\right)}{n !\left(\Phi^{\prime}(\sigma)\right)^{n}} \leqslant \max \left\{\frac{\mu\left(\sigma, F^{(k)}\right)}{k !\left(\Phi^{\prime}(\sigma)\right)^{k}}: 0 \leqslant k \leqslant N\right\}
$$

для всех $\sigma<A$ и $n \in \mathbb{Z}_{+}$.

В случае, когда $A=+\infty$, условие в) излишне.

Положим

$$
P(t)= \begin{cases}\ln \left|a_{n}\right|, & t=\lambda_{n}\left(n \in \mathbb{Z}_{+}\right), \\ -\infty, & t \in(0,+\infty) \backslash\left\{\lambda_{n}\right\},\end{cases}
$$

и

$$
Q(\sigma)=\sup \{P(t)+\sigma t: t>0\}
$$

Тогда $\ln \mu(\sigma, F)=Q(\sigma)$ и задача о поведении максимального члена ряда (1) сводится к изучению функции $Q$.

В дальнейшем считаем, что $P$ - произвольная функция, заданная на $(0,+\infty)$ и ограниченная сверху на каждом конечном интервале из $(0,+\infty)$ (она может принимать значение $-\infty$, но $P \not \equiv-\infty$; будем считать, что $P \equiv-\infty$ на $[0,1))$. Функция $Q$ назьвается двойственной с $P$ по Юнгу [3], [4, с. 86-88].

Из определения $Q$ видно, что если существует $Q\left(\sigma_{2}\right)$ и $\sigma_{1}<\sigma_{2}$, то существует $Q\left(\sigma_{1}\right)$. Поэтому либо $Q(\sigma)<+\infty$ для всех $\sigma \in \mathbb{R}$, либо $Q(\sigma)=+\infty$ для всех $\sigma \in \mathbb{R}$, либо 
существует $A \in \mathbb{R}$ такое, что $Q(\sigma)<+\infty$ для всех $\sigma<A$ и $Q(\sigma)=+\infty$ для всех $\sigma>A$. Это число $A$ будем называть абсииссой функции $Q$. Легко показать, что

$$
A=-\varlimsup_{t \rightarrow+\infty} \frac{P(t)}{t} .
$$

Действительно, если $\sigma<A$, то для $P(t) \leqslant-(A+\sigma) t / 2$ для всех $t \geqslant t_{0}(\sigma)$ и поэтому

$$
\begin{aligned}
Q(\sigma) & \leqslant \max \left\{\sup \left\{P(t)+\sigma t: 0<t \leqslant t_{0}(\sigma)\right\}, \sup \left\{-\frac{A+\sigma}{2} t+\sigma t: t \geqslant t_{0}(\sigma)\right\}\right\} \\
& \leqslant \max \left\{\sigma^{+} t_{0}(\sigma)+\sup \left\{P(t): 0<t \leqslant t_{0}(\sigma)\right\}, 1\right\}<+\infty, \quad \sigma^{+}=\max \{\sigma, 0\} .
\end{aligned}
$$

Для $\alpha \geqslant 0$ положим

$$
Q(\alpha, \sigma)=\sup \{P(t)+\alpha \ln t+\sigma t: t \geqslant 1\}
$$

Справедливость теоремы 1 легко вытекает из следующей теоремы.

Теорема 2. Пусть функиия $\Phi \in \Omega(A)$ удовлетворяет условиям а)-в) теоремы 1. Тогда для того чтобы $Q(\sigma)=O(\Phi(\sigma)), \sigma \uparrow A$, необходимо и достаточно, чтобы существовало число $B>0$ такое, что для всех $\sigma<A u \alpha \geqslant 0$

$Q(\alpha, \sigma)-\ln \Gamma(1+\alpha)-\alpha \ln \Phi^{\prime}(\sigma) \leqslant \max \left\{Q(\beta, \sigma)-\ln \Gamma(1+\beta)-\beta \ln \Phi^{\prime}(\sigma): 0 \leqslant \beta \leqslant B\right\}$.

2. Вспомогательные утверждения. Прежде всего заметим, что из определения следует, что функция $Q$ вьпукла на $(-\infty, A)$ и, значит,

$$
Q(\sigma)=Q\left(\sigma_{0}\right)+\int_{\sigma_{0}}^{\sigma} q(t) d t, \quad-\infty<\sigma_{0} \leqslant \sigma<A
$$

где $q$ - неубывающая функция на $(-\infty, A)$.

Далее, рассматривая $Q(\alpha, \sigma)$ как функцию переменных $\alpha \in[0,+\infty)$ и $\sigma \in(-\infty, A)$, легко видеть, что справедлива следующая

Лемма 1. Функиия $Q(\alpha, \sigma)$ принимает конечные значения в области $[0,+\infty) \times$ $(-\infty, A)$ и равна $+\infty$ в области $[0,+\infty) \times(A,+\infty)$. Для произвольного $\alpha \in[0,+\infty)$ функиия $Q(\alpha, \cdot)$ неубывающая $и$ выпуклая на $(-\infty, A)$, а для произвольного $\sigma \in$ $(-\infty, A)$ функиия $Q(\cdot, \sigma)$ неубывающая и выпуклая на $[0,+\infty)$.

Для $(\alpha, \sigma) \in[0,+\infty) \times(-\infty, A)$ положим

$$
q(\alpha, \sigma)=\inf _{\varepsilon>0} \sup \{t \geqslant 1: P(t)+\alpha \ln t+\sigma t \geqslant Q(\alpha, \sigma)-\varepsilon\}
$$


Лемма 2. Для фиксированного $\alpha \in[0,+\infty)$ функиия $q(\alpha, \cdot)$ неубьвающая на $(-\infty, A)$ и $Q_{\sigma}^{\prime}(\alpha, \sigma)=q(\alpha, \sigma)$ для всех $\sigma \in(-\infty, A)$ за исключением не более чем счетного множества точек, а для фиксированного $\sigma \in(-\infty, A)$ функиия $q(\cdot, \sigma)$ неубивающая на $[0,+\infty)$ и $Q_{\alpha}^{\prime}(\alpha, \sigma)=\ln q(\alpha, \sigma)$ для всех $\alpha \in[0,+\infty)$ за исключением не более чем счетного множества точек.

ДокАЗАТЕльство. Ограничимся только доказательством первого утверждения, поскольку второе утверждение доказьвается совершенно аналогично.

Покажем, что для произвольных $\varepsilon>0, \alpha \geqslant 0, \sigma \in(-\infty, A)$ множество

$$
E(\varepsilon, \alpha, \sigma)=\{t \geqslant 1:|t-q(\alpha, \sigma)|<\varepsilon, P(t)+\alpha \ln t+\sigma t \geqslant Q(\alpha, \sigma)-\varepsilon\}
$$

непустое. Действительно, зафиксируем $\varepsilon>0, \alpha \geqslant 0, \sigma<A$. Тогда существует $\delta \in(0, \varepsilon)$ такое, что

$$
|q(\alpha, \sigma)-\sup \{t \geqslant 1: P(t)+\alpha \ln t+\sigma t \geqslant Q(\alpha, \sigma)-\delta\}|<\frac{\varepsilon}{2},
$$

и существует $t_{0} \geqslant 1$ такое, что

$$
P\left(t_{0}\right)+\alpha \ln t_{0}+\sigma t_{0} \geqslant Q(\alpha, \sigma)-\delta
$$

и

$$
\left|t_{0}-\sup \{t \geqslant 1: P(t)+\alpha \ln t+\sigma t \geqslant Q(\alpha, \sigma)-\delta\}\right|<\frac{\varepsilon}{2} .
$$

Из (6)-(8) получаем

$$
\left|t_{0}-q(\alpha, \sigma)\right|<\varepsilon, \quad P\left(t_{0}\right)+\alpha \ln t_{0}+\sigma t_{0} \geqslant Q(\alpha, \sigma)-\varepsilon,
$$

т.е. $t_{0} \in E(\varepsilon, \alpha, \sigma)$ и, значит, $E(\varepsilon, \alpha, \sigma) \neq \varnothing$.

Отсюда по аксиоме выбора для каждого $\varepsilon>0$ существует функция $q_{\varepsilon}:[0,+\infty) \times$ $(-\infty, A) \rightarrow[1,+\infty)$ такая, что для всех $\alpha \in[0,+\infty)$ и $\sigma \in(-\infty, A)$

$$
Q(\alpha, \sigma) \geqslant P\left(q_{\varepsilon}(\alpha, \sigma)\right)+\alpha \ln q_{\varepsilon}(\alpha, \sigma)+\sigma q_{\varepsilon}(\alpha, \sigma) \geqslant Q(\alpha, \sigma)-\varepsilon
$$

и

$$
\left|q(\alpha, \sigma)-q_{\varepsilon}(\alpha, \sigma)\right|<\varepsilon .
$$

Пусть $\sigma_{1}, \sigma_{2} \in(-\infty, A)$. В силу определения

$$
Q\left(\alpha, \sigma_{1}\right) \geqslant P\left(q_{\varepsilon}\left(\alpha, \sigma_{2}\right)\right)+\alpha \ln q_{\varepsilon}\left(\alpha, \sigma_{2}\right)+\sigma_{1} q_{\varepsilon}\left(\alpha, \sigma_{2}\right)
$$

Из (10) имеем

$$
Q\left(\alpha, \sigma_{2}\right) \leqslant \varepsilon+P\left(q_{\varepsilon}\left(\alpha, \sigma_{2}\right)\right)+\alpha \ln q_{\varepsilon}\left(\alpha, \sigma_{2}\right)+\sigma_{2} q_{\varepsilon}\left(\alpha, \sigma_{2}\right),
$$

а из (12) и (13) получаем

$$
Q\left(\alpha, \sigma_{2}\right)-Q\left(\alpha, \sigma_{1}\right) \leqslant\left(\sigma_{2}-\sigma_{1}\right) q_{\varepsilon}\left(\alpha, \sigma_{2}\right)+\varepsilon
$$


Переходя к пределу при $\varepsilon \rightarrow 0$ и учитывая $(11)$, имеем

$$
Q\left(\alpha, \sigma_{2}\right)-Q\left(\alpha, \sigma_{1}\right) \leqslant\left(\sigma_{2}-\sigma_{1}\right) q\left(\alpha, \sigma_{2}\right)
$$

Поскольку $\sigma_{1}, \sigma_{2}$ произвольные, то, меняя их местами, получаем также неравенство

$$
Q\left(\alpha, \sigma_{1}\right)-Q\left(\alpha, \sigma_{2}\right) \leqslant\left(\sigma_{1}-\sigma_{2}\right) q\left(\alpha, \sigma_{1}\right)
$$

Пусть $\sigma_{1}<\sigma_{2}$. Тогда из (14) и (15) имеем

$$
q\left(\alpha, \sigma_{1}\right) \leqslant \frac{Q\left(\alpha, \sigma_{2}\right)-Q\left(\alpha, \sigma_{1}\right)}{\sigma_{2}-\sigma_{1}} \leqslant q\left(\alpha, \sigma_{2}\right),
$$

откуда легко следует справедливость первого утверждения леммы 2.

ЛЕмма 3. Для фиксированного $\sigma \in(-\infty, A)$ и всех $\alpha \in[0,+\infty)$ за исключением не более чем счетного множества точек выполняется неравенство

$$
Q_{\sigma}^{\prime}(\alpha, \sigma) \exp \{Q(\alpha, \sigma)\} \leqslant \exp \{Q(\alpha+1, \sigma)\}
$$

ДоКАЗАТЕЛЬСТво. Из леммы 2 следует, что

$$
Q(\alpha+1, \sigma)-Q(\alpha, \sigma)=\int_{\alpha}^{\alpha+1} \ln q(\xi, \sigma) d \xi \geqslant \ln q(\alpha, \sigma)
$$

т.е. $\exp \{Q(\alpha, \sigma)\} q(\alpha, \sigma) \leqslant \exp \{Q(\alpha+1, \sigma)\}$. Отсюда, снова учитывая лемму 2 , получаем неравенство (16).

Лемма 4. Пусть положительная ограниченная функиия $G$ задана на прямоугольнике $[0, B] \times[a, b]$ и удовлетворяет условиям:

1) для каждого $\beta \in[0, B]$ функиия $G(\beta, \cdot)$ абсолютно непрерывна на $[a, b]$;

2) для каждого $x \in[a, b]$ функиия $G(\cdot, x)$ непрерывна на $[0, B]$;

3) для каждого $\beta \in[0, B]$ и почти для всех $x \in[a, b]$

$$
G_{x}^{\prime}(\beta, x) \leqslant \varphi(x) g(x), \quad g(x)=\max \{G(\xi, x): 0 \leqslant \xi \leqslant B\},
$$

әде $\varphi$ - положсительная непрерывная на $[a, b]$ функиия.

Tогда

$$
\ln g(x) \leqslant \ln g(a)+\int_{a}^{x} \varphi(t) d t
$$


ДокАЗАТЕЛЬСТво. Пусть $\delta, \gamma \in[a, b]$. Покажем, что

$$
g(\gamma)-g(\delta) \leqslant \max \{G(\xi, \gamma)-G(\xi, \delta): 0 \leqslant \xi \leqslant B\} .
$$

Действительно, существуют $\xi_{1}, \xi_{2} \in[0, B]$ такие, что $g(\gamma)=G\left(\xi_{1}, \gamma\right), g(\delta)=G\left(\xi_{2}, \delta\right)$. Тогда $G\left(\xi_{1}, \delta\right) \leqslant G\left(\xi_{2}, \delta\right)$ и поэтому

$$
g(\gamma)-g(\delta)=G\left(\xi_{1}, \gamma\right)-G\left(\xi_{2}, \delta\right) \leqslant G\left(\xi_{1}, \gamma\right)-G\left(\xi_{1}, \delta\right),
$$

откуда следует (19).

Так как

$$
G(\beta, \gamma)-G(\beta, \delta)=\int_{\delta}^{\gamma} G_{x}^{\prime}(\beta, x) d x
$$

то в силу (17)

$$
G(\beta, \gamma)-G(\beta, \delta) \leqslant \int_{\Delta(\delta, \gamma)} \varphi(x) g(x) d x,
$$

где $\Delta(\delta, \gamma)$ - отрезок с концами $\delta, \gamma$. Отсюда в силу (19)

$$
g(\gamma)-g(\delta) \leqslant \int_{\Delta(\delta, \gamma)} \varphi(x) g(x) d x
$$

и, следовательно,

$$
|g(\gamma)-g(\delta)| \leqslant \int_{\Delta(\delta, \gamma)} \varphi(x) g(x) d x
$$

для любых $\delta, \gamma \in[a, b]$.

Из определения функции $g$ и непрерывности функции $\varphi$ следует, что функция $\varphi g$ ограничена и измерима по мере Лебега, т.е. $\varphi g$ интегрируемая на $[a, b]$. Отсюда, учитывая (20), получаем, что функция $g$ абсолютно непрерьвна на $[a, b]$. Используя абсолютную непрерьвность $g$ и неравенство

$$
g(\gamma+\Delta \gamma)-g(\gamma) \leqslant \int_{\gamma}^{\gamma+\Delta \gamma} \varphi(x) g(x) d x, \quad \Delta \gamma>0,
$$

легко получаем, что почти для всех $\gamma \in[a, b]$ вьполняется $g^{\prime}(\gamma) \leqslant \varphi(\gamma) g(\gamma)$. В силу положительности и абсолютной непрерывности функции $g$ функция $\ln g$ также абсолютно непрерьвна. Поэтому из последнего неравенства имеем

$$
\ln g(x)-\ln g(a)=\int_{a}^{x}(\ln g(t))^{\prime} d t=\int_{a}^{x} \frac{g^{\prime}(t)}{g(t)} d t \leqslant \int_{a}^{x} \varphi(t) d t .
$$

Лемма 4 доказана.

Нам понадобится также следующий результат из [5]. 
Лемма 5. Если $\Phi$ - выпуклая непрерывно дифференцируемая на $(-\infty, A)$ функиия, то для того чтобь для каждой выпуклой на $(-\infty, A)$ функиии $Q$

$$
\varlimsup_{\sigma \rightarrow A} \frac{Q(\sigma)}{\Phi(\sigma)}<+\infty \Longrightarrow \varlimsup_{\sigma \rightarrow A} \frac{Q^{\prime}(\sigma)}{\Phi^{\prime}(\sigma)}<+\infty
$$

необходимо и достаточно, чтобъ

$$
\varlimsup_{\sigma \rightarrow A} \frac{1}{\Phi^{\prime}(\sigma)} \inf \left\{\frac{\Phi(t)}{t-\sigma}: t>\sigma\right\}<+\infty .
$$

3. Доказательство теоремы 2. Предположим, что

$$
\varlimsup_{\sigma \rightarrow A} \frac{Q(\sigma)}{\Phi(\sigma)}=T<+\infty
$$

Так как

$$
\inf \left\{\frac{\Phi(t)}{t-\sigma}: t>\sigma\right\}=\frac{\Phi\left(\Psi^{-1}(\sigma)\right)}{\Psi^{-1}(\sigma)-\sigma}=\frac{\Phi\left(\Psi^{-1}(\sigma)\right)}{\Psi^{-1}(\sigma)-\Psi\left(\Psi^{-1}(\sigma)\right)}=\Phi^{\prime}\left(\Psi^{-1}(\sigma)\right),
$$

то условие а) теоремы 2 и условие (21) эквивалентные. Поэтому по лемме 5 из (5) и (22) имеем

$$
\varlimsup_{\sigma \rightarrow A} \frac{q(\sigma)}{\Phi^{\prime}(\sigma)}=T_{1}<+\infty .
$$

Пусть $h \in(0, A-\sigma)$. Тогда в силу (5)

$$
\begin{aligned}
Q(\alpha, \sigma) & =\sup \{P(t)+\alpha \ln t+(\sigma+h) t-h t: t \geqslant 1\} \\
& \leqslant Q(\sigma+h)+\sup \{\alpha \ln t-h t: t \geqslant 1\} \\
& =Q(\sigma+h)+\alpha \ln \frac{\alpha}{e}-\alpha \ln h=Q(\sigma)+\int_{\sigma}^{\sigma+h} q(t) d t+\alpha \ln \frac{\alpha}{e}-\alpha \ln h \\
& \leqslant Q(\sigma)+h q(\sigma+h)+\alpha \ln \frac{\alpha}{e}-\alpha \ln h,
\end{aligned}
$$

откуда ввиду (23) получаем

$$
Q(\alpha, \sigma) \leqslant Q(\sigma)+T_{2} h \Phi^{\prime}(\sigma+h)+\alpha \ln \frac{\alpha}{e}-\alpha \ln h .
$$

Если положим здесь $h=1 / \Phi^{\prime}(\sigma)$, то в силу условия в) $\sigma+h<A$, а в силу условия б)

$$
\begin{aligned}
& Q(\alpha, \sigma)-\ln \Gamma(1+\alpha)-\alpha \ln \Phi^{\prime}(\sigma) \\
& \quad \leqslant Q(\sigma)-\ln \Gamma(1+\alpha)+\alpha \ln \frac{\alpha}{e}+\frac{T_{2}}{\Phi^{\prime}(\sigma)} \Phi^{\prime}\left(\sigma+\frac{1}{\Phi^{\prime}(\sigma)}\right) \\
& \quad \leqslant Q(\sigma)-\frac{1}{2} \ln (2 \pi \alpha)+T_{3} \leqslant Q(\sigma)
\end{aligned}
$$


для всех $\sigma \in(-\infty, A)$ и всех $\alpha \geqslant \alpha_{0}$. Отсюда легко получаем (4).

Наоборот, из (4) имеем

$$
\frac{\exp \{Q(\alpha, \sigma)\}}{\Gamma(1+\alpha)\left(\Phi^{\prime}(\sigma)\right)^{\alpha}} \leqslant g(\sigma)=\max \left\{\frac{\exp \{Q(\beta, \sigma)\}}{\Gamma(1+\beta)\left(\Phi^{\prime}(\sigma)\right)^{\beta}}: 0 \leqslant \beta \leqslant B\right\} .
$$

Так как функция $Q(\alpha, \sigma)$ вьпуклая (лемма 1$)$ и $\Phi^{\prime \prime} \geqslant 0$, то, используя лемму 3 , для произвольных $\beta \in[0, B]$ и $\sigma \in(-\infty, A)$ за исключением не более чем счетного множества точек вьполняется неравенство

$$
\begin{aligned}
\left(\frac{\exp \{Q(\beta, \sigma)\}}{\Gamma(1+\beta)\left(\Phi^{\prime}(\sigma)\right)^{\beta}}\right)_{\sigma}^{\prime} & =\frac{\exp \{Q(\beta, \sigma)\} Q_{\sigma}^{\prime}(\beta, \sigma)}{\Gamma(1+\beta)\left(\Phi^{\prime}(\sigma)\right)^{\beta}}-\frac{\beta \exp \{Q(\beta, \sigma)\} \Phi^{\prime \prime}(\sigma)}{\Gamma(1+\beta)\left(\Phi^{\prime}(\sigma)\right)^{\beta+1}} \\
& \leqslant \frac{\exp \{Q(\beta, \sigma)\} Q_{\sigma}^{\prime}(\beta, \sigma)}{\Gamma(1+\beta)\left(\Phi^{\prime}(\sigma)\right)^{\beta}} \\
& \leqslant \max \left\{\frac{\exp \{Q(\beta, \sigma)\} Q_{\sigma}^{\prime}(\beta, \sigma)}{\Gamma(1+\beta)\left(\Phi^{\prime}(\sigma)\right)^{\beta}}: 0 \leqslant \beta \leqslant B\right\} \\
& \leqslant \max \left\{\frac{\exp \{Q(\beta+1, \sigma)\}}{\Gamma(1+\beta+1)\left(\Phi^{\prime}(\sigma)\right)^{\beta+1}} \frac{\Gamma(1+\beta+1)}{\Gamma(1+\beta)} \Phi^{\prime}(\sigma): 0 \leqslant \beta \leqslant B\right\} \\
& \leqslant(B+1) \Phi^{\prime}(\sigma) g(\sigma) .
\end{aligned}
$$

Отсюда по лемме 4 на каждом конечном интервале $[a, \sigma) \subset(-\infty, \sigma)$

$$
\begin{aligned}
\ln g(\sigma) & \leqslant \ln g(a)+\int_{a}^{\sigma}(B+1) \Phi^{\prime}(t) d t \\
& =\ln g(a)+(B+1)(\Phi(\sigma)-\Phi(a))=C+(B+1) \Phi(\sigma)
\end{aligned}
$$

где $C \equiv$ const. Так как $Q(\sigma) \leqslant \ln g(\sigma)$, то тем самым теорема 2 полностью доказана.

\section{СПИСОК ЦИТИРОВАННОЙ ЛИТЕРАТУРЫ}

[1] Шеремета М. Н. О последовательностях максимальньх членов и центральньх показателей производных рядов Дирихле // Матем. заметки. 1998. Т. 63. № 3. С. 457-467.

[2] Шеремета М. Н., Федыняк С. И. О производной ряда Дирихле // Сиб. матем. ж. 1998. T. 39. № 1. C. 206-223.

[3] Young W. H. On classes of summable functions and their Fourier series // Proc. Roy. Soc. (A). 1912. V. 87. P. 225-229.

[4] Евграфов М. А. Асимптотические оценки и целые функции. М.: Физматгиз, 1962.

[5] Братищев А. В. Об обрашении правила Лопиталя // Механика сплошной среды. Ростов-на-Дону: Изд. РГУ, 1985. С. 28-42. 